\title{
QualiPRO: Eine Internetplattform zur Erleichterung der Studienteilnahme für Prüfärzte, Prüfzentren und Studienzentralen
}

\author{
Kristina Ihrig Nicola Gökbuget \\ Medizinische Klinik II, Klinikum der Goethe-Universität, Frankfurt a.M., Deutschland
}

\author{
Schlüsselwörter \\ Online-Datenbank · Eignungsnachweise · Prüfer · \\ Lebenslauf · Ethikkommission
}

\section{Zusammenfassung}

Investigator-initiierte Studien (IIT) stellen deutschlandweit den Versorgungsstandard in der Hämatologie und Onkologie dar und zielen zugleich auf stetige Therapieoptimierung ab. Mit der 12. Arzneimittelgesetz(AMG)-Novelle wurden die regulatorischen Vorgaben für IIT denen von Zulassungsstudien angeglichen. Insbesondere die Beantragung von Ethikvoten stellt eine große Herausforderung dar, da gegebenenfalls für bis zu 51 Ethikkommissionen umfangreiche Antragsunterlagen bereitgestellt werden müssen. Auch im Jahr 2012, nach Änderung des AMG, sind diese Prozesse komplex, wobei die Verantwortung nun auf den Prüfer am Zentrum übertragen worden ist. Aus diesen Gründen wurde die Online-Datenbank "QualiPRO» entwickelt, kostenfrei und einfach zugänglich für Prüfer, ihre Studienteams und Studienzentralen von Multicenterstudiengruppen. QualiPRO vereinfacht den Prozess, einen vollständigen, aktuellen Lebenslauf (Curriculum Vitae (CV)) für die Teilnahme an klinischen Studien sowie andere benötigte Dokumente bereitzustellen. Der Inhalt und die Architektur der Datenbank berücksichtigen die Empfehlungen des Arbeitskreises medizinischer Ethikkommissionen und die Good Clinical Practice (GCP) der International Conference on Harmonisation (ICH). Nach Registrierung der eigenen Abteilung und der Mitgliedschaft der Kollegen können Daten eingegeben, bearbeitet und ein CV mit ausführlicher Studienaktivität leicht erzeugt werden. CV, GCP-Zertifikate, Zulassungsbescheinigungen und andere Dokumente stehen nach dem Upload zentral den Teammitgliedern und den Studienzentralen von Therapieoptimierungsstudien zur Verfügung, sofern eine Einwilligung der Studienteilnahme vorliegt. Außerdem liefert QualiPRO Übersichten für Gruppenleitungen über die Studienaktivität und den Einsatz des Studienpersonals sowie dessen Trainingsstand.

\author{
Keywords \\ Online database $\cdot$ Suitability $\cdot$ Investigator \\ Curriculum vitae $\cdot$ Ethical review board
}

\section{Summary}

QualiPRO: Online Database to Facilitate Study Participation for Investigators, Study Sites and Coordinating Centers Academic non-commercial trials are of substantial relevance for both patient care and progress in clinical research. Since the 12th amendment of the German drug law, applications for the initiation of clinical trials at the ethical review boards are much more cost- and time-intensive, particularly for multicenter therapy optimization trials (TOS). To activate a trial, for, e.g., 51 ethical review boards, current curricula vitae (CVs) and certificates on good clinical practice (GCP) and regulations have to be provided for all investigators. After the new amendment of the German drug law in 2012, the process remains complex while the responsibility of the team eligibility has now been transferred to the main investigator at the study site. Therefore, the online database 'QualiPRO' was developed, free of charge and widely accessible for the investigators, their clinical trial units and the coordinating centers of the TOS. Its features ease the process to generate and provide data on the clinical trial activities of any investigator and team member. The database content and architecture follows ethical review board recommendations and the Good Clinical Practice (GCP) of the International Conference on Harmonisation (ICH). After registration of the unit and membership of the team, study staff members are able to enter and edit data and to print a 'trial' CV. CVs, GCP certificates, licences to practice medicine and more can be uploaded, and, after consent of the investigators, are available to the coordinating centers of TOS. In addition, QualiPRO is an instrument for directors of hospital departments or group leaders to efficiently collect and locally display data on their study activities and on the training status of their staff.

\begin{tabular}{ll}
\hline KARGER & @ 2013 S. Karger GmbH, Freiburg \\
Fax +49 761 452 07 14 & 0378-584X/13/3614-0036\$38.00/0 \\
Information@Karger.com & Accessible online at: \\
www.karger.com & www.karger.com/onk
\end{tabular}

Dr. med. Nicola Gökbuget 
QualifikationsPROfile) bezeichnete Plattform fertiggestellt und steht gleichermaßen Studienärzten, Studienpersonal und Investigator-initiierte Studien (IIT) stellen deutschlandweit den Versorgungsstandard in der Onkologie dar und zielen zugleich auf stetige Therapieoptimierung ab. Eine Vielzahl dieser Studien wird von Studiengruppen durchgeführt, die in indikationsbezogene Netzwerke integriert sind. In den Kompetenznetzen Leukämien (KNL) bzw. maligne Lymphome (KML) werden seit über 30 Jahren erfolgreich Therapieoptimierungen unter Beteiligung von über 100 Zentren durchgeführt.

Mit der 12. Arzneimittelgesetz(AMG)-Novelle [1] wurden die regulatorischen Vorgaben für IIT jedoch denen von $\mathrm{Zu}$ lassungsstudien angeglichen und die Durchführung dieser Studien erschwert.

Es wurde daher im KNL eine Arbeitsgruppe gegründet, die das Ziel verfolgt, effiziente Lösungen für alle Studiengruppen zu erarbeiten und der Gefährdung der mittlerweile etablierten Studienkultur akademischer Studien entgegenzusteuern.

Die Arbeitsgruppe «AG Studienzentralen» setzt sich aus den ärztlichen Leitern der Studienzentralen der großen Multicenterstudiengruppen und den Mitarbeitern der jeweiligen Studienzentralen mit Erfahrung in studienbezogenen Fachgebieten aus den KNL und KML zusammen. Die Mitarbeiter der AG sind auch in anderen Gremien sowohl auf internationaler als auch nationaler Ebene aktiv. In den Studiengruppen wurde bereits früh die Belastung anerkannt, die aufgrund der 12. AMG-Novelle insbesondere im Bezug auf die Beantragung von Ethikvoten auf die Studienzentralen übertragen worden ist (siehe Beitrag N. Gökbuget in diesem Sonderheft).

\section{Entstehungsgeschichte des Projekts}

Bereits im Jahr 2006 wurde im Arbeitskreis Arzneimittelgesetz (AK AMG) der Deutschen Gesellschaft für Hämatologie und Medizinische Onkologie (DGHO) e.V. die Idee eines zentralen Systems für die Erfassung von Lebensläufen (Curriculum Vitae (CV)) und weiterer Unterlagen zur Vereinfachung der Arbeitsabläufe in multizentrisch aktiven Studienzentralen vorgestellt und für sinnvoll befunden. In der AG Studienzentralen des KNL wurde daraufhin ein Konzept für eine internetbasierte quelloffene (open-source) WebDaten- und Dokumentebank zur elektronischen Erfassung und Sammlung der benötigten Dokumente entwickelt. Bei der Festlegung der Formularinhalte und der Datenbankarchitektur wurden Vorgaben der Verordnung «Gute klinische Praxis» (GCP-V) [2], Vorgaben des Arbeitskreises der medizinischen Ethikkommissionen (AK EK) und die internationale Leitlinie ICH-GCP (International Conference on Harmonisation of Technical Requirements for Registration of Pharmaceuticals for Human Use - Good Clinical Practice) berücksichtigt. Im Jahr 2011 wurde die als QualiPRO (für

\section{Was ist das Ziel von QualiPRO?}

Oberstes Ziel des Projekts ist die Reduktion des Zeitaufwands für administrative Prozesse, wie das zeitaufwendige Erstellen und Aktualisieren eines fachlichen GCP-konformen $\mathrm{CV}$ und das Bereitstellen der Eignungsnachweise durch Prüfer und Studienpersonal sowie das Einholen der Eignungsnachweise für die Studienteilnahme durch die zuständige Studienzentrale. Zusätzlich ergeben sich weitere Vorteile, z.B. eine abteilungsspezifische Darstellung der Studienaktivität mit den ihnen zugeordneten Prüfärzten und Studienpersonal.

\section{QualiPRO - Features}

QualiPRO ist eine Website mit integrierter Daten- und Dokumentebank und Software-Funktionen, um ausgewählte Studiendaten, abteilungsspezifische Studienaktivitäten und Dokumente für Studienbeteiligungen korrekt, vollständig und effizient zu sammeln (Abb. 1). «Abteilungsspezifisch» bedeutet, dass jede in QualiPRO angemeldete Abteilung als eine geschlossene Einheit für das System zugelassen ist. Dadurch sind die eigenen Studienaktivitäten und Dokumente nicht von anderen klinischen Abteilungen einsehbar.

Zusätzlich bietet das System Funktionen, um einen eigenen «fachlichen GCP-konformen Lebenslauf» zu erstellen. «Fachlicher $\mathrm{CV}$ » bedeutet, dass Daten rein persönlicher Natur, z.B. das Geburtsdatum, nicht erfasst werden. Der CV enthält vielmehr Informationen zur Studieneignung; dazu zählen neben der medizinischen Ausbildung der Prüfer auch studienbezogene Erfahrungen und Fortbildungen in GCP [3].

Die Hauptfunktionen von QualiPRO werden über vier verschiedene Menüs bedient, die im Folgenden dargestellt werden.

\section{Menü «Meine Daten»}

Dieses Menü beinhaltet den Hauptdatensatz für den eigenen CV. Dieser Datensatz ist personenbezogen und kann ausschließlich von der Person bearbeitet werden, die sich mit ihrem persönlichen Benutzernamen und Passwort einloggt. Zur homogenen und korrekten Eingabe erläutern Hilfetexte die jeweiligen Datenfelder.

Kerninhalte dieses Formulars sind:

- fachbezogene persönliche Angaben (z.B. Titel)

- medizinische Qualifikationen

- studienspezifische Qualifikationen: Beschreibung von GCP-Trainings (Dokumente werden eingescannt und im Menü «Dokumente» hochgeladen) usw.

- Studienerfahrungen: Liste der bisherigen Studienerfahrungen 
Abb. 1. QualiPRO-Website und QualiPROFeatures.

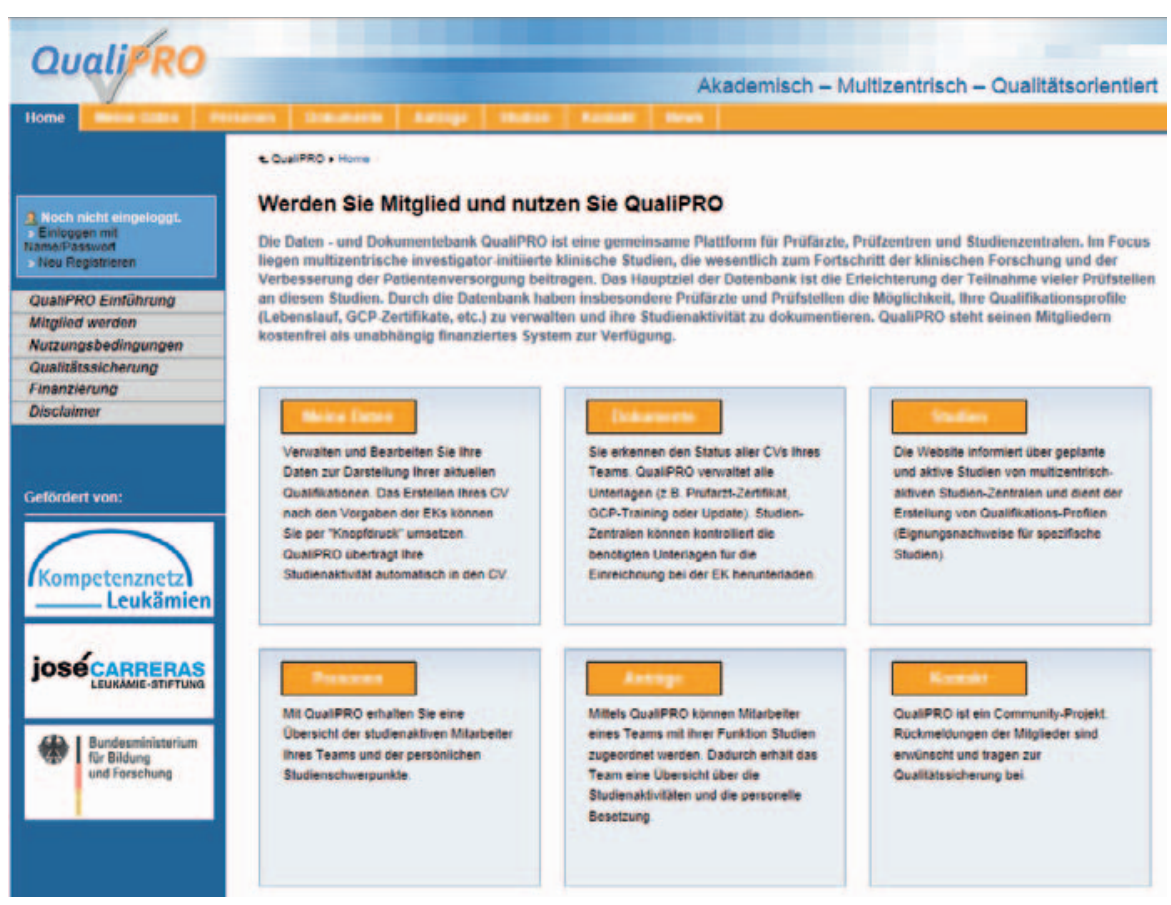

Das System sieht eine Übersetzung des CV ins Englische für Standardangaben vor, sodass die Voraussetzungen für eine deutsche und eine englischsprachige Version gegeben sind. Um die englische Version zu erstellen, müssen nur wenige Freitextfelder ins Englische übersetzt werden.

Nach Datenspeicherung wird der CV als Word-Dokument generiert. Durch die Ausgabe der Daten in eine WordDatei kann der CV noch zu diesem Zeitpunkt bei Bedarf individuell an eigene Bedürfnisse angepasst werden. Das finalisierte, unterschriebene Dokument wird eingescannt und dann über das Menü «Dokumente» hochgeladen (siehe Abb. 2 für ein Muster eines fachlichen GCP-konformen CV (QualiPRO)).

\section{Menü «Dokumente»}

Das System zielt in erster Linie auf die Sammlung von Dokumenten im Zusammenhang mit Studien ab; es können aber auch andere Unterlagen eingestellt werden.

Die Dokumente werden, sortiert nach Dokumentenart, übersichtlich angezeigt und können von allen Mitgliedern der Abteilung heruntergeladen werden. Dadurch wird ein zentraler Ort für die Qualifikationsnachweise von Prüfern und Studienpersonal in der Abteilung geschaffen, der für alle Teammitglieder zugänglich ist. So werden im Falle von geplanten Studienbeteiligungen rasch alle aktuellen, vollständigen Dokumente heruntergeladen und z.B. an die Studienzentrale, eine Clinical Research Organization (CRO) oder die Ethikkommission weitergeleitet.

$\mathrm{Zu}$ den in QualiPRO eingestellten Dokumenten gehören:

- fachliche GCP-konforme CV

- GCP-Zertifikate, Prüfarztkurse und andere Dokumente zum Nachweis der Studienqualifikation
- Sonstiges, z.B. inhaltliche Fortbildungen zu Erkrankungen Um die Aktualität der Lebensläufe zu gewährleisten, werden die CV in diesem Menü farblich markiert und zu gegebener Zeit wird eine automatische Erinnerungsmail initialisiert. Aktuelle CV werden grün im Menü «Dokumente» des Systems QualiPRO dargestellt. Nach etwa einem Jahr erhält das Mitglied die Aufforderung, seinen CV neu zu erstellen. Sollte dies nicht geschehen, wird der CV zuerst gelb, dann rot als veraltet im System markiert.

\section{Menü «Anträge»}

Die Hauptfunktion dieses Menüs besteht für Abteilungen, Prüfer und Studienpersonal in der Zuordnung der Kollegen und Kolleginnen zu einer Studie, die in der Prüfstelle durchgeführt wird - mit Angabe der Funktion der Person in dieser Studie.

Zur Auswahl für Funktionen stehen z.B. Leiter der klinischen Prüfung (LKP), Prüfer, Prüfer (Stellvertreter), Mitglied der Prüfgruppe, Hauptprüfer, Hauptprüfer (Stellvertreter), Studienassistenz, Koordination, Projektleitung. Die Zuordnung selbst kann durch jedes Mitglied für sich selbst erfolgen oder aber durch die für die Studie zuständige Studienassistenz - je nach Regelung in der Abteilung.

Mit dieser Zuordnung werden relevante Daten (z.B. European Union Drug Regulating Authorities Clinical Trial (EudraCT)-Nummer, wissenschaftlicher Titel, Phase der Studie usw.) in den Datensatz für den CV übertragen, sodass ein Ausdruck im CV zu den Studienaktivitäten erfolgt. Der CV wird immer über das Menü «Meine Daten» generiert, der Ausdruck der Studienaktivität erfolgt im CV priorisiert nach Funktion in der Studie und zeitlich nach Beginn der Studie. Zugleich besteht aber auch immer die Möglichkeit, Angaben zu Studien im Menü «Meine Daten» zu machen, die nicht die- 

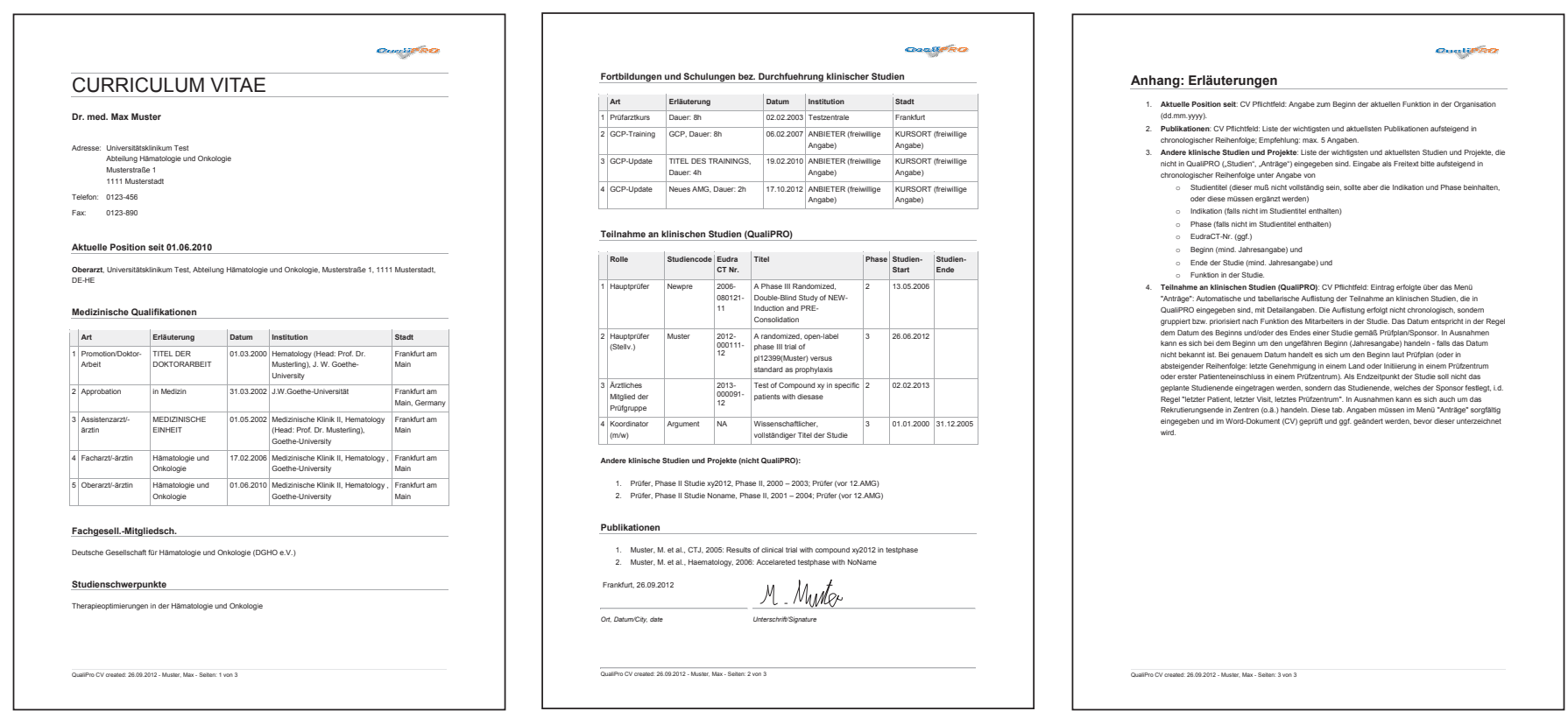

Abb. 2. Muster für einen fachlichen GCP-konformen CV (QualiPRO).

Tab. 1. Vorteile von QualiPRO für Prüfärzte und Studienpersonal

Tab. 2. Vorteile von QualiPRO für Abteilungsleitungen und Hauptprüfer
Standardisierte Form und Inhalte von CV

Berücksichtigung der Vorgaben des Arbeitskreises der medizinischen Ethikkommissionen Ausführliche Hilfetexte für die korrekte Eingabe

Automatisierte Generierung neuer Studienbeteiligungen in den CV

Übersichtliche Verwaltung eigener Unterlagen

Erinnerung an CV-Update

$\mathrm{CV}$ in deutscher und englischer Sprache

Zentrale Ablage und komfortabler Download aller notwendigen Eignungsnachweise des Studienteams

Übersicht der Studienaktivitäten des Teams (inkl. Personen, die den Studien als Prüfer/ Studienpersonal zugeordnet sind unter Angabe ihrer Funktion in der Studie)

Übersicht des Fortbildungsstands des Teams im Bezug auf Studien

Einfache Verwaltung der Qualifikationsprofile (GCP-Zertifikate, CV usw.)

Übersichtliche Anzeige der Aktualität und Art der Dokumente (insbesondere Aktualität der CV) Beschleunigung und Vereinfachung der Studienteilnahme als Prüfstelle ser «automatischen» Zuordnung unterliegen. Für Prüfärzte hat das System an dieser Stelle den wesentlichen Vorteil, dass sie nicht ständig selbst ihre Studienaktivitäten im Lebenslauf aktualisieren müssen. Diese Funktion von QualiPRO kann nach bisheriger Erfahrung sinnvoll genutzt werden, wenn dieser Prozess klar geregelt ist.

\section{Menü «Studien»}

Dieses Menü wird genutzt, um neue Studien in das System einzugeben. Es müssen nur wenige Daten für Browseranzeige, Studienaktivität und CV-Generierung aufgenommen werden. Für teilnehmende Abteilungen sind auch die Studien ersichtlich, die an anderen Standorten schon eingegeben wurden. Aktuell beinhaltet die Datenbank bereits einige Hundert Studien, da hämatologische Studien des KNL mit Beginn der
Datenerfassung in QualiPRO (2009) eingegeben sind. Die Anzeige dieser über 180 Studien erfolgt gemäß dem medizinischen Fachgebiet in der Gruppe «Innere Hämatologie/Onkologie» und alphabetisch nach dem vom Sponsor vergebenen Kurztitel. Neben hämatologischen Studien sind bereits auch eine Anzahl anderer Studien, z.B. Studien des Fachgebiets «Kinder mit Schwerpunkt Hämatologie/Onkologie», erfasst.

\section{Welche Vorteile bietet QualiPRO?}

Vorteile ergeben sich für Prüfer, Studienpersonal, Studienkoordinatoren, Mitarbeiter im Qualitätsmanagement, Abteilungsleitungen und Studienzentralen. Die wesentlichen Vorteile sind in den Tabellen 1 und 2 dargestellt. 


\section{Wie kann QualiPRO genutzt werden?}

QualiPRO steht seinen Mitgliedern kostenfrei als unabhängig finanziertes System zur Verfügung.

Der erste Schritt ist die Registrierung der Abteilung (= Organisation). Dieser Schritt ist notwendig, um die «in sich geschlossene Abteilung in QualiPRO» für die Nutzung festzulegen. Antragsteller ist in der Regel der Abteilungsleiter unter Mithilfe eines/r koordinierenden Mitarbeiters/in. Der 1-seitige Antrag wird von der Website heruntergeladen und an die QualiPRO-Zentrale gefaxt (www.quali-pro.de > Mitglied werden $>$ Für Kliniken und Praxen $>$ Registrierung der Organisation). Für alle weiteren Schritte werden ausführliche Anleitungen zur Verfügung gestellt. Die QualiPRO-Zentrale unterstützt alle Antragsteller und ihre Mitarbeiter bei der individuellen Umsetzung des Systems im eigenen Haus.

\section{QualiPRO mit Blick auf die AMG-Gesetzesänderung (Oktober 2012)}

Aufgrund zahlreicher kritischer Rückmeldungen seitens unabhängig forschender Ärzte, unter anderem auch durch die DGHO, wurde Anfang Juli 2012 eine erneute Gesetzesänderung des AMG bzw. der GCP-V zunächst im Bundestag [4] und am 21. September 2012 auch im Bundesrat beschlossen $[5,6]$ : Nun wird unter anderem die Prüfarztverantwortung auf nur einen Prüfer und einen Stellvertreter am Zentrum übertragen. Bei entsprechender Umsetzung durch die Ethikkommission sind dann Einzelnachweise für die Eignung weiterer ärztlicher Kollegen und Kolleginnen der Prüfgruppe nicht mehr den Antragsunterlagen für die Ethikkommission beizufügen. Zugleich wird jedoch der festgelegte Prüfer als Leiter des Prüferteams am Zentrum mehr in die Verantwortung genommen, mit dem Ergebnis, dass auch die Qualifikation des Teams nun stärker in seinem Verantwortungsbereich liegt. Daher bietet QualiPRO auch in dieser Konstellation große Vorteile. So können Prüfer jederzeit einen aktuellen Überblick über die Qualifikation ihrer Gruppe bewahren und nach außen dokumentieren. Prüfer haben die Möglichkeit, ihre CV und Qualifikationsnachweise im System komfortabel zu pflegen. Alle anderen Ärzte können jederzeit ihre Dokumente nutzen, wenn sie eine Prüferfunktion übernehmen.

\section{Förderung}

Mit Unterstützung durch das BMBF (Fördernummer 01G19971) und die Deutsche José Carreras Leukämie-Stiftung (Fördernummer H09/01f).

\section{Disclosure Statement}

Die Autoren haben für diese Arbeit keine Interessenkonflikte offenzulegen.

\section{Literatur}

1 Arzneimittelgesetz in der Fassung der Bekanntmachung vom 12. Dezember 2005 (BGBl. I S. 3394), zuletzt geändert durch Artikel 1 der Verordnung vom 16. Juli 2012 (BGBl. I S. 1534).

2 GCP-Verordnung vom 9. August 2004 (BGBl. I S 2081), zuletzt geändert durch Artikel 4 der Verordnung vom 3. November 2006 (BGBl. I S. 2523).
3 Empfehlung zur Bewertung der Qualifikation von Prüfern und Geeignetheit von Prüfstellen durch Ethik-Kommissionen bei klinischen Prüfungen nach AMG. Dtsch Arztebl 2010;107:A-48, B-40, C-40.

4 Drucksache 17/9341, Gesetzentwurf der Bundesregierung: Entwurf eines Zweiten Gesetzes zur Änderung arzneimittelrechtlicher und anderer Vorschriften (Dt. Bundestag, 18.04.2012).
5 Drucksache 17/10156, Beschlussempfehlung und Bericht des Ausschusses für Gesundheit (14. Ausschuss): Zweites Gesetz zur Änderung arzneimittelrechtlicher und anderer Vorschriften (27.06.2012).

6 Drucksache 487/12, Beschluss des Bundesrates: Zweites Gesetz zur Änderung arzneimittelrechtlicher und anderer Vorschriften (21.09.2012). 